\title{
SUPERIOR VENA CAVA SYNDROME AFTER HEART TRANSPLANTATION: PERCUTANEOUS TREATMENT OF A COMPLICATION OF BICAVAL ANASTOMOSES
}

\author{
Daniel Y. Sze, MD, $\mathrm{PhD}^{\mathrm{a}}$ \\ Robert C. Robbins, $\mathrm{MD}^{\mathrm{b}}$ \\ Charles P. Semba, MD ${ }^{\mathrm{a}}$ \\ Mahmood K. Razavi, MD \\ Michael D. Dake, MD
}

\begin{abstract}
Objectives: Our objectives were (1) to investigate the incidence and cause of symptomatic superior vena caval anastomotic stenosis and central venous thrombosis in patients receiving heart or heart-lung transplantation and (2) to explore percutaneous methods of thrombolysis and endoluminal intervention to treat these complications. Methods: Review of 1016 cases revealed three cases of superior vena cava syndrome. Anatomy, surgical technique, and medical risk factors were examined. Percutaneous treatments, including urokinase thrombolysis, mechanical thrombolysis, balloon angioplasty, and stent placement, were attempted. Results: All three of these patients underwent transplantation by means of the bicaval anastomotic technique. In addition, the diameters of the donor and recipient cavae were grossly mismatched in all three. Stenoses in all three patients were successfully treated percutaneously with balloon angioplasty and stent placement. Treatment of the accompanying large-volume thrombosis was problematic in these patients, and two had hemorrhagic complications of urokinase thrombolysis. A mechanical thrombolysis device was used successfully in the third patient. Conclusions: Anastomotic stricture and central venous thrombosis is an uncommon complication of the bicaval anastomotic technique of heart and heart-lung transplantation. Discrepancy between donor and recipient caval diameters appears to be the major risk factor. Endoluminal thrombolysis and stenting provides rapid and enduring relief of symptoms and precludes repeat sternotomy, cardiopulmonary bypass, and general anesthesia. (J Thorac Cardiovasc Surg 1998; 116:253-61)
\end{abstract}

0 the basis of early success in animal models, orthotopic cardiac transplantation in human subjects has been customarily performed by means of the biatrial anastomotic technique first described by Shumway and coworkers. ${ }^{1,2}$ This technique was designed to simplify the vena caval and pulmonary venous anastomoses. Although its overall success has been unequivocally proven, a number of noteworthy complications occur with regular frequency. Most consequentially, a significant proportion of these patients have short-term

From the Division of Cardiovascular and Interventional Radiology $^{\mathrm{a}}$ and Department of Cardiothoracic Surgery, ${ }^{\mathrm{b}}$ Stanford University Medical Center, Stanford, Calif.

Received for publication Feb. 3, 1998; accepted for publication March 8, 1998.

Address for reprints: Daniel Y. Sze, MD, PhD, Division of Cardiovascular and Interventional Radiology, Stanford University Medical Center, H3630, Stanford, CA 94305-5450.

Copyright (C) 1998 by Mosby, Inc.

$0022-5223 / 98 \$ 5.00+0 \quad \mathbf{1 2 / 1 / 9 0 1 8 5}$ and long-term arrhythmias, asynchronous and compromised atrial contraction, atrioventricular valve incompetence, and other hemodynamically significant sequelae resulting from sinoatrial node and conduction pathway disruption and altered atrial physiology. ${ }^{3-5}$

More recently, an alternative technique involving complete resection of the recipient's right atrium and primary anastomoses of the superior and inferior venae cavae (SVC and IVC, respectively) has been shown to reduce the incidence and severity of many of these complications. ${ }^{6-8}$ These advantages are also applicable to heart-lung transplantation. In addition, complete excision of the donor and recipient hearts facilitates "domino" procedures. 9 Numerous centers have adopted the bicaval anastomotic technique as a new standard, improving the likelihood of rapid and complete recovery of normal physiologic function. However, the necessity of primary end-to-end anastomoses of the donor and recipient cavae presents special surgical challenges. 
Anastomotic strictures are a widely known and well-studied phenomenon in the arena of cardiac and vascular surgery, and even large central veins such as the venae cavae are susceptible to this complication.

We identified three cases of symptomatic SVC syndrome in patients who underwent heart or heartlung transplantation with bicaval anastomoses. All three patients had anastomotic stenoses as well as extensive central venous thrombosis. To date, obstructive complications of only the SVC have been encountered, and no instances of IVC obstruction have been detected. Although hemodynamically significant stenoses of the SVC are rare, we are able to identify the likely causes and thus propose special surgical precautions in susceptible patients.

In addition, we describe nonsurgical percutaneous methods of treating these SVC obstructions using interventional radiologic techniques. These techniques preclude the need for repeat sternotomy or thoracotomy, cardiopulmonary bypass, and even general anesthesia. Percutaneous techniques, which include angioplasty, stents, thrombolysis, and thrombectomy, have long been applied successfully to the arterial vascular system. More recently, the applicability to the venous system, particularly in deep vein thrombosis of the upper and lower extremities, is being established. ${ }^{10}$ The success of percutaneous treatment of central venous obstruction or occlusion varies depending on anatomic considerations and on cause of the thrombosis. Our initial results of percutaneous treatment of anastomotic strictures of the SVC have been extremely encouraging, and as is generally true for treatment of SVC obstruction from other causes, clinical response has been immediate, dramatic, and durable. $^{11}$

\section{Clinical summaries}

PATIENT 1. A newborn girl was diagnosed with complex congenital cardiac anomalies, including double-inlet left ventricle, transposition of the great vessels, large trabecular ventricular septal defect, small muscular ventricular septal defect, and hypoplastic and dysplastic pulmonic and mitral valves. At 1 day of age, she underwent an uncomplicated balloon septostomy at the time of diagnostic cardiac catheterization. At 7 months of age, she underwent a modified Glenn procedure, and at 3 years 9 months of age, she underwent a modified Fontan procedure. Although her hemodynamic function improved with each of these procedures, post-Fon- tan protein-losing enteropathy with biopsy-proven lymphangiectasis developed. ${ }^{12}$

When she was 5 years of age, an orthotopic heart transplantation was performed, involving takedown of the modified Glenn shunt and excision of the modified Fontan repair. End-to-end anastomoses were performed for the pulmonary artery, aorta, SVC, and IVC, with a single left atrial button used for a rim anastomosis. Seven days before the transplantation, a central venous catheter inserted in the left internal jugular vein became occluded and was removed, and a Hickman catheter (C.R. Bard, Inc., Salt Lake City, Utah) was placed into the right external jugular vein.

Immediate postoperative recovery proceeded without major complications. At 2 months, the patient was noted to have increasing facial swelling and occasional oxygen desaturations. A transesophageal echocardiogram performed on postoperative day 62 showed thrombus in the SVC, at which time the Hickman catheter was removed and a right femoral line was placed. Unfortunately, the patient also passed melenic stool and had an episode of hematemesis on postoperative day 62. Endoscopy revealed a small but nonbleeding antral ulcer.

On postoperative day 70 , the patient underwent cardiac catheterization, including pulmonary angiography, but no evidence of pulmonary emboli was found. However, from the femoral approach, interrogation of the SVC revealed complete occlusion. The occlusion was traversed with a wire and catheter, and injection of contrast material peripheral to the occlusion demonstrated collateral drainage through mediastinal and pericardial veins, communicating with the pulmonary veins and left atrium (Fig. 1). Balloon dilatation of the SVC was performed with a $6 \mathrm{~mm}$ and then an $8 \mathrm{~mm}$ diameter balloon, but normal flow was not reestablished. Despite the patient's recent history of gastrointestinal bleeding, a cautious course of thrombolysis was attempted. A coaxial multiple side-hole EDM infusion catheter (Mallinckrodt Medical, Inc., St. Louis, Mo.) and a Sos infusion wire (Cook Inc., Bloomington, Ind.) were placed directly into the thrombus, and urokinase (Abbott Laboratories, North Chicago, Ill.), 80,000 IU/hr, was infused for a total of 36 hours. During this period, venography was repeated twice, demonstrating progressive recanalization of the obstruction.

After 36 hours of lysis, the patient had a recurrent gastrointestinal hemorrhage as evidenced by melena 

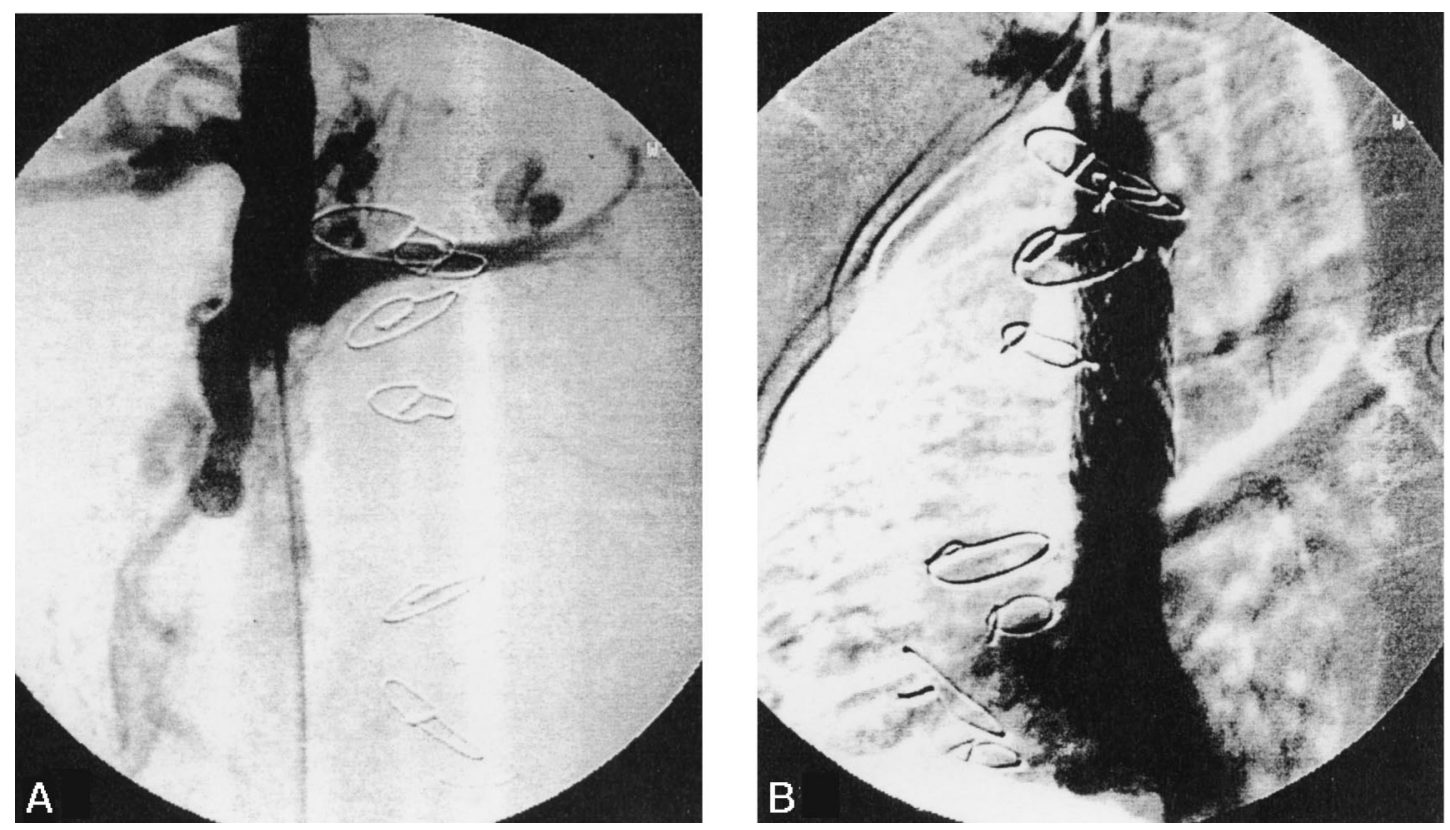

Fig. 1. Venograms of a 5-year old girl 70 days after heart transplantation for post-Fontan protein-losing enteropathy. A, Superior venacavogram at time of presentation, demonstrating complete occlusion and slow drainage through collateral vessels. B, Reestablishment of rapid, in-line flow after catheter-directed urokinase thrombolysis and placement of a stent.

and a nine-point drop in hematocrit value to $23 \%$. Urokinase was discontinued, and a transfusion was given. Another venogram showed only partial reestablishment of flow, and balloon dilatation was again performed. An area of persistent stenosis measuring less than $3 \mathrm{~mm}$ in diameter was seen even after balloon venoplasty. Finally, a Palmaz 308 stent (Johnson \& Johnson, Warren, N.J.) was deployed and dilated to $12 \mathrm{~mm}$ diameter. This maneuver established rapid antegrade flow and an angiographically normal-appearing luminal contour.

The patient's facial swelling resolved within 48 hours and has not recurred in 32 months of followup. During a myocardial biopsy performed 18 months after treatment, venography confirmed rapid antegrade flow, and pressure measurements revealed no gradient. Incidentally, a right iliofemoral deep vein thrombosis developed during the period of thrombolysis despite therapeutic levels of heparin. Because of the patient's history of multiple venous thromboses, a hematologic workup was pursued, revealing heterozygous factor V Leiden mutation. However, the patient has done well without long-term anticoagulation therapy.
PATIENT 2. A 47-year old woman had progressive right heart failure, hypoxemia, and polycythemia from Eisenmenger's syndrome, despite apparently successful surgical repair of an atrial septal defect at the age of 36 years. The patient underwent heartlung transplantation with bicaval anastomoses and recovered uneventfully until postoperative day 4 , when transient hypotension and hypoxemia developed. Transthoracic echocardiogram and bronchoscopy were unrevealing. On postoperative day 8 , the patient was noted to have distended forehead and neck veins and moderate plethora of the head and neck, but the central venous pressure, measured through a left subclavian catheter whose tip was in the SVC, was only $4 \mathrm{~mm} \mathrm{Hg}$.

Venography was performed through a right basilic vein catheter, introduced under ultrasonic guidance. This demonstrated complete occlusion of the SVC, with nonocclusive thrombus extending into the right brachiocephalic vein to the level of the internal jugular/subclavian confluence (Fig. 2). Additional access was obtained from the right common femoral vein, and the SVC occlusion was easily traversed with wire and catheter. Venography of the left subclavian vein demonstrated nonocclusive 

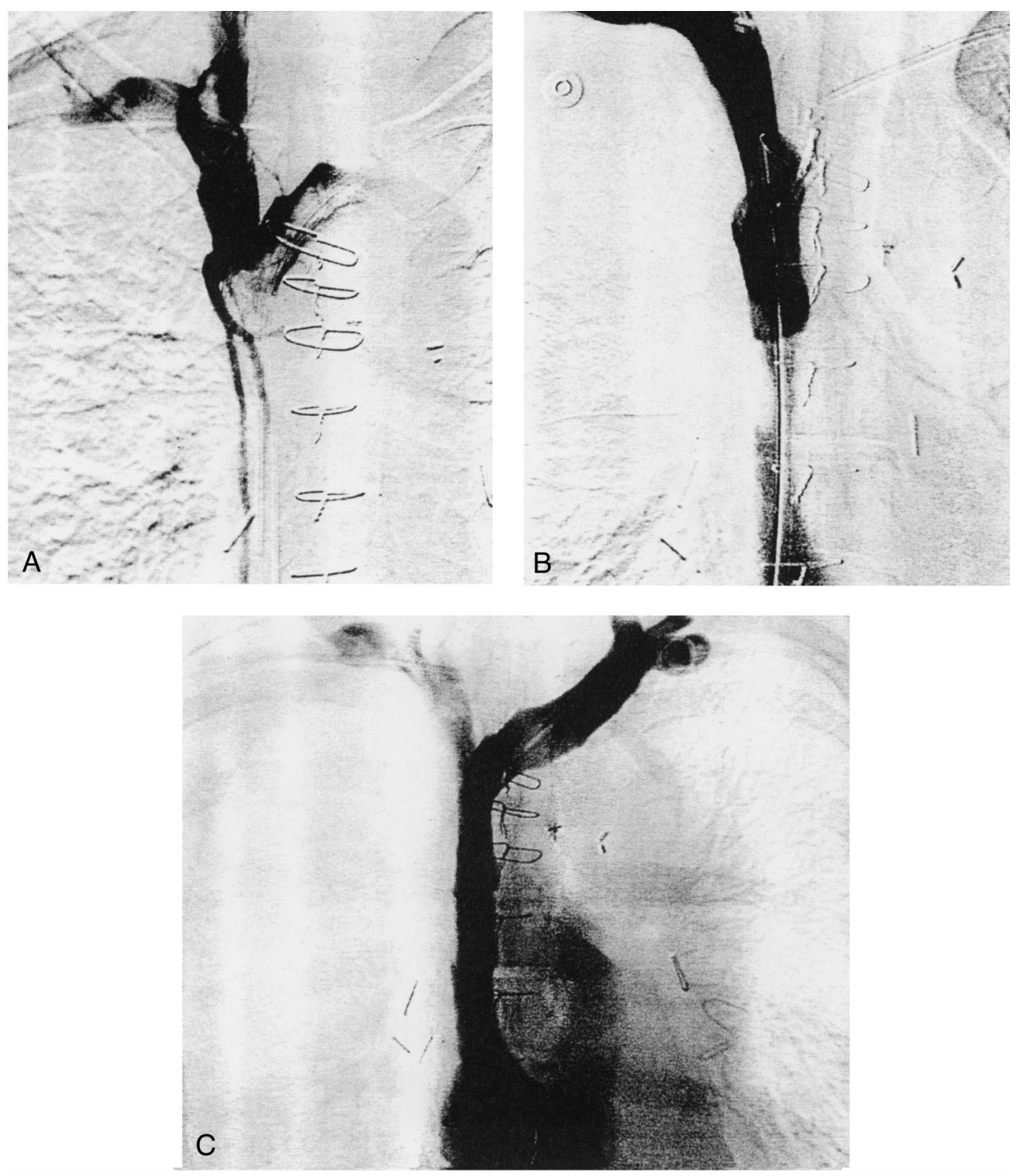

Fig. 2. Venograms of a 47-year-old woman 8 days after heart-lung transplantation for Eisenmenger's syndrome and atrial septal defect. A, Superior venacavogram demonstrating extensive thrombosis of central veins and minimal collateral drainage through mediastinal veins. B, Thrombolysis revealed underlying stenosis, which responded poorly to balloon angioplasty. C, Restoration of flow after placement of a stent. Note right hemothorax, a complication of thrombolysis.

thrombus in the left axillary, subclavian, internal jugular, and brachiocephalic veins.

Thrombolysis was commenced via a Mewissen multiple side-hole catheter (Meditech/Boston Scientific Corp., Watertown, Mass.), introduced from the right basilic vein and spanning the right-sided thrombus, and a Sos infusion wire via the femoral vein spanning the left-sided thrombus. A total urokinase dose of 170,000 IU/hr was administered for 45 hours. Thrombolytic progress was checked by venography twice and showed partial recanalization. Thrombolysis was discontinued when the patient's hematocrit value dropped 10 points in 10 hours, and a chest radiograph revealed a new, large right 
pleural effusion. Approximately $800 \mathrm{ml}$ of fresh hemorrhagic effusion was drained, and the patient was given a transfusion.

Even after complete lysis of the thrombus, a persistent stenosis of the SVC was evident on venography, $15 \mathrm{~mm}$ in length and $3 \mathrm{~mm}$ in smallest diameter. Initial balloon venoplasty did not identify a tight waist, but the elastic recoil of the region prevented maintenance of a sufficiently large channel after dilatation. A Palmaz 308 stent was then deployed and dilated to $14 \mathrm{~mm}$ luminal diameter. Post-stenting venography demonstrated rapid flow and a smooth luminal contour. The patient's venous distention and plethora resolved within 24 hours. The woman was discharged on postoperative day 14 . Although no follow-up imaging has been performed, subsequent myocardial biopsies via a right internal jugular vein approach have been without incident, and the patient remains free of symptoms in 30 months of follow-up.

Patient 3. A 37-year old man with cystic fibrosis had oxygen-dependent end-stage respiratory failure. His medical history was significant for chronic lung colonization, leading to cerebral abscesses and chronic seizure disorder at the age of 20 years and bleeding complications of laparoscopic cholecystectomy and endoscopic sinus surgery, both necessitating reoperation, at the age of 35 years. The patient underwent a domino heart-lung transplantation with bicaval anastomoses and required reexploration of the right chest wall on postoperative day 1 for bleeding from collateral vessels.

On postoperative day 9, massive edema of the head and neck developed. A transthoracic echocardiogram revealed no significant cardiac disease, but echogenic material in the internal and external jugular veins was identified. Bilateral upper extremity venograms, performed on postoperative day 11 by means of basilic vein catheters, revealed complete thrombosis of bilateral subclavian, internal jugular, and brachiocephalic veins, as well as of the SVC (Fig. 3). Only minimal collateral vessels were seen in the thoracic wall, suggesting acute thrombosis. Of note, the patient had a ported central venous catheter (Bard Access, Salt Lake City, Utah) that had been inserted through the right internal jugular vein several years before the transplant procedure. A venogram of the IVC was performed from a femoral access and showed no abnormalities.

Because of the patient's recent operations, history of bleeding complications, and history of intracerebral lesions, thrombolysis was deemed unacceptably risky. Instead, mechanical thrombolysis was performed with an Angiojet device (Possis Medical Inc., Minneapolis, Minn.). ${ }^{13}$ This $5 \mathrm{~F}$ wire-guided catheter device uses high-pressure jets of saline solution to develop a Venturi suction effect. Soft thrombus can be removed without the use of thrombolytic agents and with only minor risk of downstream embolization. This device was used extensively in this patient's thrombosed vessels, reestablishing antegrade flow. However, a critical stenosis of the SVC persisted, resulting in drainage via retrograde flow in the azygos vein.

From the left brachial vein access, the tip of the central venous line in the right internal jugular vein was snared and pulled out of the SVC into the left brachiocephalic vein. A total of three overlapping Palmaz 308 stents were then deployed in the SVC stenosis and dilated to $14 \mathrm{~mm}$ diameter. The central line tip was then repositioned within the stents. Final venography demonstrated wide patency and rapid antegrade flow. The ported central line was removed the next day. The patient's head and neck edema decreased immediately after stenting and resolved completely within 4 days (Fig. 4). For the remainder of the hospitalization, the patient required special attention to gastrointestinal and upper airway complications of cystic fibrosis and underwent a third thoracotomy for left hemothorax on postoperative day 26 . He was discharged on day 40 and has remained free of symptoms referable to SVC obstruction in 12 months of follow-up.

\section{Discussion}

Orthotopic cardiac transplantation with the use of bicaval anastomoses was first proposed by Webb, Howard, and Neely ${ }^{14}$ in 1959. However, preference for complete excision of the recipient heart and bicaval anastomoses for orthotopic heart and heartlung transplantation emerged only with the advent of domino procedures 30 years later. Even in nondomino situations, certain advantages of this technique have made it the procedure of choice at many institutions. In a prospective, randomized trial in 75 patients comparing the biatrial technique to the bicaval technique in cardiac transplantation, El Gamel and associates ${ }^{6}$ reported numerous significant hemodynamic differences. Patients receiving bicaval anastomoses had lower right atrial pressures, improved right atrial contractility, fewer atrial tachyarrhythmias and decreased need for temporary pacing, lesser incidence of mitral valve incompetence, 

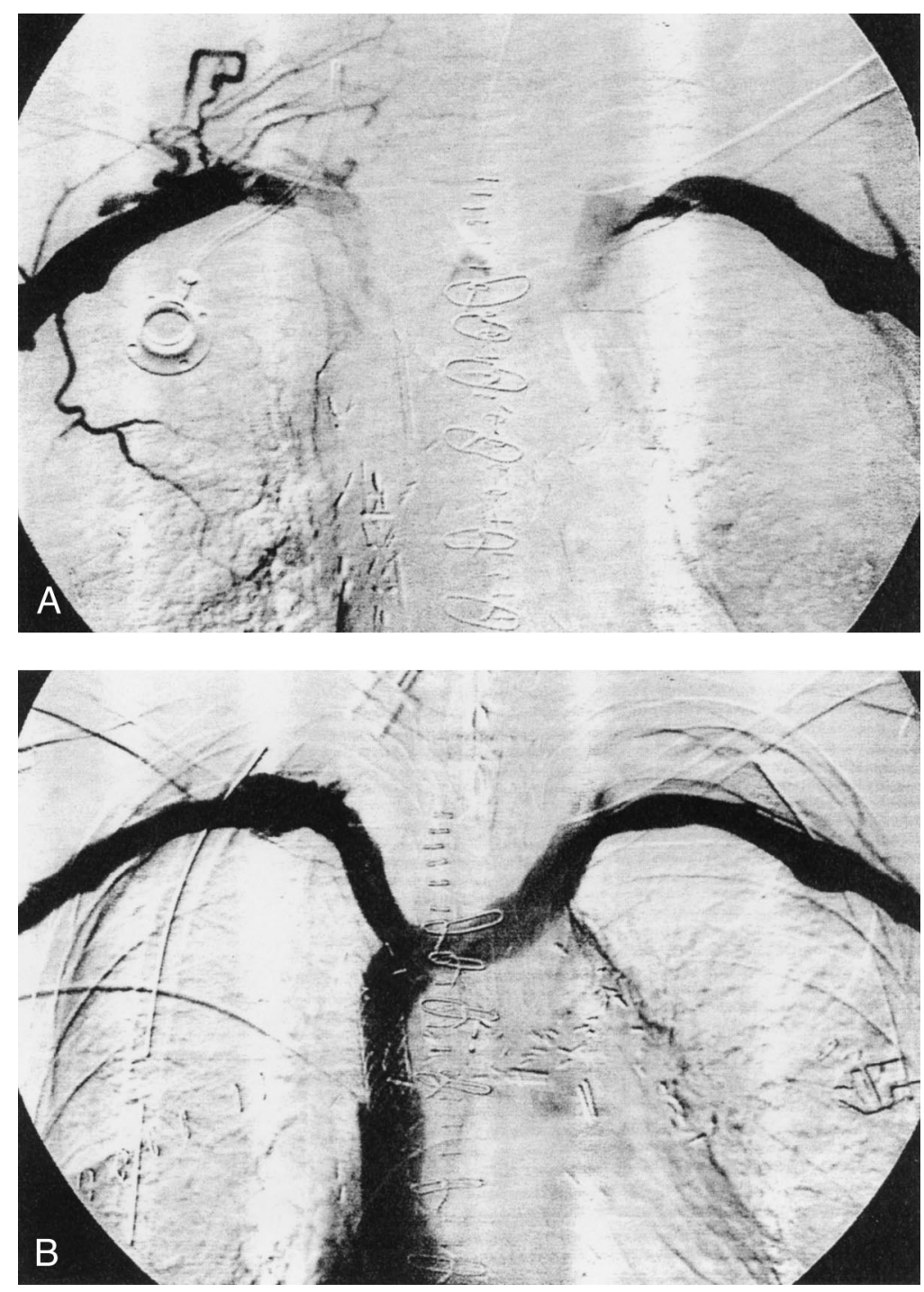

Fig. 3. Venograms of a 37-year-old man 11 days after domino heart-lung transplantation for cystic fibrosis. A, Simultaneous bilateral upper extremity venograms demonstrating stasis and central thrombosis with minimal formation of collateral drainage. B, Repeat venograms after mechanical thrombolysis and placement of stents. Inflow of unopacified blood from jugular veins causes dilutional effect on brachiocephalic opacification.

overall shorter hospitalizations, and overall increased short-term survival.

Similarly, in another prospective, randomized trial of 78 patients, Deleuze and coworkers ${ }^{7}$ found decreased atrial arrhythmias and increased early recovery of sinus rhythm, increased cardiac index, decreased right atrial and left atrial areas, decreased tricuspid valve incompetence, and decreased postoperative pulmonary arterial pressures in patients receiving bicaval anastomoses. Other retrospective studies have also found improved left atrial func- tion, ${ }^{5}$ improved exercise tolerance and capacity, ${ }^{4}$ but no significant difference in ventricular function ${ }^{15}$ in patients receiving bicaval anastomoses.

The data for short-term efficacy seem compelling, but the data for long-term efficacy are still pending. Specific disadvantages of the bicaval anastomotic technique are primarily technical and are infrequently discussed. Of the few reported complications associated exclusively with bicaval anastomoses, SVC stenosis has been reported only twice. Blanche and coworkers ${ }^{16}$ reported a case of symp- 

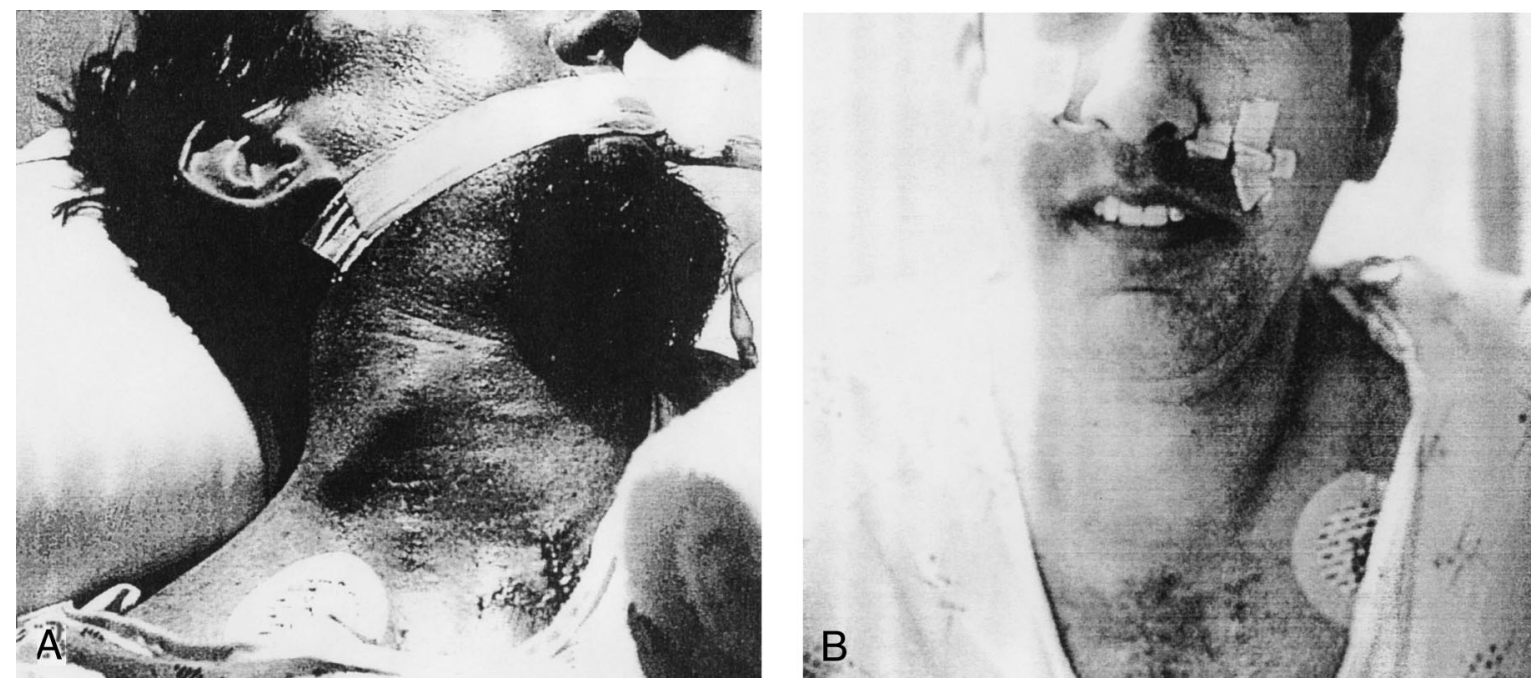

Fig. 4. A, Massive plethora of head, neck, and bilateral upper extremities, and distended external jugular vein caused by SVC syndrome in patient 3. B, Patient's appearance before discharge 28 days after thrombolysis. Plethora completely resolved 4 days after thrombolysis and stent placement. (The patient shaved off his beard.)

tomatic SVC stenosis in a 62-year-old patient who underwent orthotopic cardiac transplantation for end-stage ischemic cardiomyopathy. No evidence of thrombosis of the central veins was seen. This stenosis required surgical repair 1 month after the original transplantation, consisting of the interposition of a $20 \mathrm{~mm}$ cryopreserved aortic allograft. General anesthesia, repeat sternotomy, and normothermic cardiopulmonary bypass were required. The repair was successful, and the patient remained free of symptoms in 32 months of follow-up.

In another report, Pedrazzini and colleagues ${ }^{17}$ described an SVC anastomotic stricture impeding transjugular myocardial biopsy on postoperative day 14 in a 52-year-old man who required transplantation for end-stage dilated cardiomyopathy. Although a pressure gradient of $10 \mathrm{~mm} \mathrm{Hg}$ was measured across this stricture, the patient remained free of symptoms with no evidence of thrombosis. The stenosis was treated percutaneously with balloon angioplasty, effectively reducing the gradient to $4 \mathrm{~mm} \mathrm{Hg}$. The patient remained free of signs or symptoms of venous congestion, despite a moderate stenosis by angiographic appearance and by pressure measurements.

Our data confirm that stricture of the SVC anastomosis is very uncommon. At Stanford University, from January 1968 to December 1997, 933 orthotopic heart transplantations have been performed on 866 patients. From March 1981 to September
1997, 154 heart-lung transplantations have been performed on 150 patients. Since the first bicaval anastomosis done in 1992, 124 heart transplantations have been performed by means of the biatrial technique, 75 heart transplantations have been performed by the bicaval technique, and all 52 heartlung transplantations have involved use of the bicaval technique. In the past year, 35 of 46 procedures (76\%) performed by six surgeons involved bicaval anastomoses. Our incidence of anastomotic stricture in patients undergoing bicaval anastomosis is thus 1 of $75(1.3 \%)$ for heart transplants, 2 of $52(3.8 \%)$ for heart-lung transplants, and 3 of $127(2.4 \%)$ overall.

The major technical difficulty and the most likely significant risk factor for development of an anastomotic stricture is donor-recipient mismatch of caval diameter. A notable proportion of transplant recipients have had a history of chronic elevated central venous pressures, tricuspid valve incompetence, anomalous venous drainage, or a combination thereof. Over time, these hemodynamic abnormalities may lead to dilatation of the central systemic venous drainage, including the coronary sinus, azygos vein, IVC, and SVC. All three of our cases, as well as the case reported by Pedrazzini and the case reported by Blanche, involved gross mismatches in SVC size.

Technical possibilities for performing a mismatched anastomosis include transection of the donor cava at a bias, using a patch to enlarge the donor cava, and using a gusset or wedge resection to 
taper the recipient cava. Trento, Blanche, and coworkers ${ }^{8}$ have developed a method preserving 1 to 2 $\mathrm{mm}$ of a right atrial cuff in the recipient and using the azygos vein to enlarge the donor caval stump. They adopted this modification after encountering their one recognized case of anastomotic stricture, and subsequently they have not detected any additional strictures in about 90 patients. Increased technical complexity and manipulation near the anastomosis, however, carries the theoretical risk of disrupting the sinus node or its arterial supply. ${ }^{18}$ Preserving a right atrial cuff also complicates domino donation.

Stricture formation in the caudal anastomosis has never been reported, and gross mismatch of IVC sizes is almost never seen. The encasement of the intrahepatic segment, as well as the larger average size of the IVC, may provide some protection against dilatation of the native IVC. However, in orthotopic liver transplantation, stenoses of the IVC anastomoses do occur. These stenoses may be treated by percutaneous methods ${ }^{19-21}$ or avoided by using a "piggyback" technique that precludes caval transection and anastomosis. ${ }^{22}$

Percutaneous treatment of venous obstruction has mirrored the success of treatment of the arterial system. Thrombolysis, angioplasty, and stenting of SVC obstruction has been performed since $1987 .^{23}$ A large majority of these patients have an underlying malignant tumor that may directly invade, encase, or compress the cava, or they have mediastinal lymphadenopathy that does the same. Other causes of venous obstruction include mediastinal fibrosis such as from granulomatous disease or radiation therapy. A large proportion of these patients have the added risk factor of central venous lines or pacemaker leads, which can cause or augment an erosive, phlebitic, or fibrotic reaction, particularly at the mobile tip of a long-standing catheter. ${ }^{24}$

In all three of our cases, caval stenosis was severe enough to result in central venous thrombosis. Catheter-directed thrombolysis of deep vein thrombosis has been increasingly accepted, especially in cases in which an underlying obstruction can be identified and treated. ${ }^{25}$ Highest local concentrations of thrombolytic agents are achieved with infusion catheters and wires that can be buried directly into the thrombus. Local bleeding complications occur in up to $12 \%$ of cases, most frequently at the site of venous access. ${ }^{26}$ Despite the short serum half-life of thrombolytic agents (10 to 20 minutes for urokinase, 4 minutes for recombinant tissue-type plasminogen activator $^{27}$ ), systemic distribution of these agents also introduces the risk of remote bleeding complications. Commonly encountered contraindications to thrombolytic therapy include recent operation (within 10 to 20 days), recent gastrointestinal or other bleeding, intracranial neoplasm, cerebrovascular accident, particularly hemorrhagic stroke, and concomitant coagulopathy. In two of our three patients, contraindications existed (recent operation, recent gastrointestinal hemorrhage), but surgical reexploration was considered even riskier than a trial of thrombolysis. Both of these patients had significant bleeding complications (hemothorax, recurrent gastrointestinal hemorrhage) that resolved when urokinase was discontinued, but both patients required transfusions.

Our third patient had multiple contraindications to thrombolysis, including history of intracerebral abscesses, history of postoperative bleeding complications, and recent operation (posttransplantation day 9). Fortunately, a number of new mechanical thrombectomy devices that macerate and aspirate thrombi are now available. These include the Amplatz thrombectomy device (Microvena, White Bear Lake, Minn.) and the Angiojet device (Possis Medical Inc., Minneapolis, Minn.). These devices use mechanical methods to pulverize and aspirate thrombus, precluding the need for thrombolytic pharmaceuticals. Both of these devices were originally designed for use in hemodialysis fistulas, but applications elsewhere, both in arterial and in venous systems, are being explored. We present an example of an especially appropriate application of this technology in a patient who was not a candidate for biochemical thrombolysis, who was at high risk for surgical reexploration, and who had an enormous thrombus load large enough to be life-threatening if embolized into the pulmonary circulation. Further development of these technologies may find wide applicability in difficult situations such as postoperative and posttraumatic deep vein thrombosis.

\section{REFERENCES}

1. Lower R, Shumway N. Studies on the orthotopic homotransplantation of the canine heart. Surg Forum 1960;11:18-9.

2. Shumway N, Lower R, Stofer R. Transplantation of the heart. Adv Surg 1966;2:265-84.

3. Lauerma K, Harjula A, Jarvinen V, Kupari M, Keto P. Assessment of right and left atrial function in patients with transplanted hearts with the use of magnetic resonance imaging. J Heart Lung Transplant 1996;15:360-7.

4. Leyh R, Jahnke A, Kraatz E, Sievers H. Cardiovascular 
dynamics and dimensions after bicaval and standard cardiac transplantation. Ann Thorac Surg 1995;59:1495-500.

5. Freimark D, Silverman J, Aleksic I, Crues J, Blanche C, Trento A, et al. Atrial emptying with orthotopic heart transplantation using bicaval and pulmonary venous anastomoses: a magnetic resonance imaging study. J Am Coll Cardiol 1995;25:932-6.

6. El Gamel A, Yonan NA, Grant S, Deiraniya AK, Rahman AN, Sarsam M, et al. Orthotopic cardiac transplantation: a comparison of standard and bicaval Wythenshawe techniques. J Thorac Cardiovasc Surg 1995;109:721-30.

7. Deleuze P, Benvenuti C, Mazzucotelli J, Perdrix C, Le Besnerais P, Mourtada A, et al. Orthotopic cardiac transplantation with direct caval anastomosis: Is it the optimal procedure? J Thorac Cardiovasc Surg 1995;109:731-7.

8. Trento A, Takkenberg J, Czer L, Blanche C, Nessim S, Cohen M, et al. Clinical experience with one hundred consecutive patients undergoing orthotopic heart transplantation with bicaval and pulmonary venous anastomoses. J Thorac Cardiovasc Surg 1996;112:1496-503.

9. Baumgartner WA, Traill TA, Cameron DE, Fonger JD, Birenbaum IB, Reitz BA. Unique aspects of heart and lung transplantation exhibited in the domino-donor operation. JAMA 1989;261:3121-5.

10. Semba C, Dake M. Catheter-directed thrombolysis for iliofemoral venous thrombosis. Sem Vasc Surg 1996;9:26-33.

11. Kee S, Kinoshita L, Razavi M, Nyman U, Semba C, Dake M. Superior vena cava syndrome: treatment with catheter-directed thrombolysis and endovascular stent placement. Radiology 1998;206:187-93.

12. Feldt R, Driscoll D, Offord K, Cha R, Perrault J, Schaff H, et al. Protein-losing enteropathy after the Fontan operation. J Thorac Cardiovasc Surg 1996;112:672-80.

13. Wagner H, Muller-Hulsbeck S, Pitton M, Weiss W, Wess M. Rapid thrombectomy with a hydrodynamic catheter: results from a prospective, multicenter trial. Radiology 1997;205: 675-81.

14. Webb W, Howard H, Neely W. Practical method of homologous cardiac transplantation. J Thorac Surg 1959; 37:361-6.

15. Bizouarn P, Treilhaud M, Portier D, Train M, Michaud JL. Right ventricular function early after total or standard orthotopic heart transplantation. Ann Thorac Surg 1994; 57:183-7.
16. Blanche C, Tsai T, Czer L, Valenza M, Aleksic I, Trento A. Superior vena cava stenosis after orthotopic heart transplantation: complication of an alternative surgical technique. Cardiovasc Surg 1995;3:549-2.

17. Pedrazzini G, Mohacsi P, Meyer B, Carrel T, Meier B. Percutaneous transvenous angioplasty of a stenosed bicaval anastomosis after orthotopic cardiac transplantation. J Thorac Cardiovasc Surg 1996;112:1667-9.

18. Rosado L, Huston C, Sethi G, Copeland J. Sinoatrial node dysfunction in recipients of domino heart transplants: complication of a surgical harvesting technique. J Heart Lung Transplant 1992;11:1078-82.

19. Pfammatter T, Williams DM, Lane KL, Campbell DA Jr, Cho KJ. Suprahepatic caval anastomotic stenosis complicating orthotopic liver transplantation: treatment with percutaneous transluminal angioplasty, Wallstent placement, or both. AJR Am J Roentgenol 1997;168:477-80.

20. Zajko AB, Sheng R, Bron K, Reyes J, Nour B, Tzakis A. Percutaneous transluminal angioplasty of venous anastomotic stenoses complicating liver transplantation: intermediate-term results. J Vasc Interv Radiol 1994;5:121-6.

21. Simo G, Echenagusia A, Camunez F, Quevedo P, Calleja IJ, Ferreiroa JP, et al. Stenosis of the inferior vena cava after liver transplantation: treatment with Gianturco expandable metallic stents. Cardiovasc Intervent Radiol 1995;18:212-6.

22. Tzakis A, Todo S, Starzl T. Orthotopic liver transplantation with preservation of the inferior vena cava. Ann Surg 1989; 210:649-52.

23. Rosch J, Bedell JE, Putnam J, Antonovic R, Uchida B. Gianturco expandable wire stents in the treatment of superior vena cava syndrome recurring after maximum-tolerance radiation. Cancer 1987;60:1243-6.

24. Molinari PS, Belani KG, Buckley JJ. Delayed hydrothorax following percutaneous central venous cannulation. Acta Anaesthesiol Scand 1984;28:493-6.

25. Semba C, Dake M. Iliofemoral deep venous thrombosis: aggressive therapy with catheter-directed thrombolysis. Radiology 1994;191:487-94.

26. Dake M, Semba C. Thrombolytic therapy in venous occlusive disease. J Vasc Intervent Radiol 1995;6:73s-7s.

27. McEvoy G. American Hospital Formulary Service, Drug Information 1997: American Society of Health-System Pharmacists, 1997. 